\title{
Scaling up: understanding movement from individual differences to population-level dispersal
}

\author{
Allan H. Edelsparre ${ }^{1,3}$, Trevor J. Hefley ${ }^{4}$, Marco A. Rodríguez ${ }^{5}$, Mark J. Fitzpatrick ${ }^{1,2,3}$ and Marla B. \\ Sokolowski ${ }^{1,2,6}$ \\ ${ }^{1}$ Department of Ecology \& Evolution, University of Toronto, Toronto ON, M5S 3B2, Canada \\ ${ }^{2}$ Department of Cell \& Systems Biology, University of Toronto ON, M5S 3G5, Canada \\ ${ }^{3}$ Integrative Behaviour and Neuroscience Group, Department of Biological Sciences, University of Toronto Scarborough, \\ Toronto ON, M1C 1A4, Canada \\ ${ }^{4}$ Department of Statistics, Kansas State University, Manhattan KS, 66506, USA \\ ${ }^{5}$ Départment des sciences de l'environnement, Université du Québec à Trois-Rivières,C.P. 500, Trois-Rivières, Québec \\ G9A 5H7, Canada \\ ${ }^{6}$ Child and Brain Development Program, Canadian Institute for Advanced Research (CIFAR), Toronto, Ontario, M5G 1M1, \\ Canada
}

\begin{abstract}
Dispersal is fundamental to life on our planet. Dispersal facilitates colonization of continents and islands. Dispersal mediates gene flow among populations, and influences the rate of spread of invasive species. Theory suggests that individuals consistently differ in dispersal propensity, however determining the relative contributions of environmental factors to individual and population-level dispersal, represent a major challenge to understand the spread of organisms. To address this, we conducted a field experiment using Drosophila melanogaster. As proxies for individuals with different dispersal propensities, we used wildtype strains of flies with natural variants of the foraging gene, known to influence dispersal in laboratory and field experiments. These included flies with for $^{\mathrm{s}}$ alleles known to be less dispersive, flies with the $f o r^{\mathrm{R}}$ alleles which are more dispersive flies as well as an outbred population established from field collected flies. We released approximately 6000 flies of each strain in an experimental arena $(100 \mathrm{~m} \times 100 \mathrm{~m})$ in the field and our recaptures were used to determine dispersal of flies over time. To estimate environmental effects on dispersal, we measured temperature, wind direction and wind speed. Using partial-differential equations we combined ecological diffusion with advection to estimate dispersal rates and responses to wind. We found that temperature effects elicited a similar response in high and low dispersal lab strains with dispersal rate increasing with temperature most rapidly at temperatures above $18^{\circ} \mathrm{C}$. This was in contrast to outbred flies which remained unresponsive to temperature changes. We also detected a response to wind with advection rates increasing linearly with wind speed for all flies in general. Our results suggest that response to temperature and wind can minimize known differences in behavioural predispositions to disperse. Our results also suggest that the direction and magnitude of wind may play a key role in the colonization and distribution of fly populations. Our findings therefore have implications for forecasting the spread of pests and invasive species as well as pathogens and vectors of disease. Our findings further contribute to the understanding of how the environment can modify behavioural predispositions and to influence population-level dispersal in fly populations in particular and insect species in general.
\end{abstract}

Keywords: Movement ecology; dispersal; behaviour; individual variation; ecological diffusion; advection; foraging gene; Drosophila melanogaster. 
Dispersal plays a fundamental role in the evolutionary and ecological processes that govern life on our planet. From an evolutionary perspective, dispersal influences range expansion and rates of diversity (Bocxlaer et al. 2010) as well as adaptive radiations from mainland continents to islands. An example of the latter is the dispersal of anole lizards across the Greater Antilles that has facilitated the repeated evolution of similar ecomorphs on separate islands (Losos et al. 1998). Additionally, the evolution of

Darwin's finches and Hawaiian honeycreepers involved a single dispersal event from the mainland that facilitated the subsequent evolution of many different forms across the archipelagos (Grant 1981; Freed et al. 1987). From an ecological perspective, dispersal influences rates of birth, death and immigration among populations and communities (Hanski and Gilpin 1991). Dispersal mediates gene flow among populations, and is an important consideration in studies of urbanized and fragmented landscapes (Cote et al. 2017, Edelsparre et al. 2018). Finally, dispersal can also influence the rate and severity of the spread of pathogens as well as invasive species (Kot et al. 1996).

Individuals within a population can disproportionally influence dispersal. Invasive cane toads from older established areas move slower and have reduced reproductive rates relative to individuals at the leading front of the invasion (Phillips 2009) which have longer legs, move faster and in straighter paths (Phillips et al. 2006). Increased dispersal rates of individuals which are at the leading front of invasions have also been observed in butterflies (Hill et al. 1999), aphids (Lombeart et al. 2014), and fish (MylesGonzalez et al. 2015). Understanding how individual variation contributes to population dispersal can have important implications for our ability to model and predict population level dispersal. A lack of understanding of the factors that affect dispersal can result in an underestimation of the spread of invasive species and the capacity for species to reclaim or colonize new habitats (Kot et al. 1996;

74 Saastamoinen et al. 2018). This, in turn, limits our ability to make accurate ecological forecasts (Clarke 
et al. 2001), which affects our ability to manage the effect of spread of invasive species on ecosystems that are being invaded or colonized (Hastings et al. 2005; Gomez-Uchida et al. 2018).

Elucidating the underlying factors that contribute to dispersal is a major challenge for the field of movement ecology (Nathan et al. 2008; Cote et al. 2017). Several morphological, physiological, and behavioural traits are linked with variation in dispersal. For example, insect wing dimorphisms that affect dispersal ability are common (e.g. pea aphid: Zera and Deno 1997; rice plant hoppers: Brisson 2010). Behavioural traits have also been linked with dispersal in a wide range of animal taxa, including birds, fishes, and lizards (Réale et al. 2007; Cote et al. 2017). Individuals have been shown to differ in their dispersal propensity depending on whether they are aggressive (Duckworth and Badyaev 2007), are risk takers (Edelsparre et al. 2013), or are sociable (Cote and Clobert 2007). In rare cases, genes that underlie the link between these behaviours and dispersal have been identified (Korsten et al. 2013; Edelsparre et al. 2014) offering unique insights into the potential mechanisms contributing to individual differences in dispersal. However, how such factors play out in nature to influence dispersal at the population-level is poorly understood (Gurarie et al. 2009).

There are three main hypotheses through which population-level variation in dispersal might arise. First, individuals within populations can differ in their predispositions to disperse if genetic differences between individuals affect dispersal directly or via differences in morphology, physiology and/or behaviour, such as those mentioned above (Sastaamoinen et al. 2018). Under this hypothesis we would expect populations to display consistent individual differences in dispersal across multiple environmental contexts (changes in climate and/or landscape). Such consistent differences could exist even if groups or individuals with different dispersal predispositions responded similarly (parallel responses) and/or differently (divergent responses) to environmental change. Second, variation in dispersal could arise largely in response to environmental factors. This could be if dispersal 
predispositions are absent or if the environmental effects are strong enough to minimize dispersal

101 predispositions. Under this scenario we would expect to see a general population response to the

102 environment and with changes in the environment driving changes in dispersal (each disperser is a

103 random draw from the population). Finally, variation in dispersal could arise through a combination of

104 the above possibilities, including conditions ranging between individual (e.g. dispersal predispositions)

105 and environmentally driven dispersal. Detecting complex relationships between key factors such as

106 those outlined above would require the implementation of dynamic models that are capable of

107 estimating the relative contribution of each factor on the movement process (Hefley et al. 2017; Hooten

108 and Hefley 2019).

109

110 In the following study we set out to address these three hypotheses by combining individual-level

111 predictors and environmental data in dynamic models designed to assess the relationship between each

112 factor over time. In our experiment, we used the common fruit fly, Drosophila melanogaster, a

113 convenient organism to model dispersal in general and to investigate individual-level predictors of

114 dispersal in particular. To accomplish this we first produced a large outbred study population from field

115 collected flies. Secondly, we incorporated individual-level predictors of dispersal by using Drosophila

116 melanogaster that carry variants of a gene known to influence differences in the propensity of the adult

117 fly to disperse (Edelsparre et al. 2014; Edelsparre et al. 2018). This particular Drosophila system

118 consists of two strains of flies that differ in several movement related behaviours both as larvae and

119 adults and these differences are mediated by natural variation in the foraging (for) gene (Osborne et al.

120 1987; de Belle et al. 1993; Pereira and Sokolowski 1993; Edelsparre et al. 2014). Individuals that carry

$121 f^{\mathrm{R}}{ }^{\mathrm{R}}$ alleles (rovers) are active foragers as larvae and more dispersive as adults while individuals that

122 carry for ${ }^{\mathrm{s}}$ alleles (sitters) tend to be less active foragers as larvae and less dispersive as adults. Recent

123 laboratory experiments demonstrated environmentally dependent plasticity in dispersal propensity of

124 the rover and sitter variants of the foraging gene (Anreiter and Sokolowski 2019, Edelsparre et al. 
125

2020). Consequently, the rover and sitter strains were used to examine how different dispersal propensities may interact with the environment to produce population-level dispersal and the outbred population was used to examine how a population with multiple dispersers respond to the environment.

Temperature and wind are two important environmental factors that influence insect activity and movement in nature (Glick 1942; Taylor 1963; McManus 1988). Work on several insect species suggests that there are critical temperatures below which insects will not initiate dispersal (Taylor 1963) and even lower temperatures beyond which insects are not able to sustain flight (Cockbain 1961). In general, insect dispersal will increase with temperature, likely with some optimal temperature range conducive to movement. However, whether such a response can be linked with dispersal predispositions remain largely unexplored. Although, wind direction and speed are thought to be critical to insect flight the behavioural response to wind is likely not a simple one (McManus 1988). Since the early 1920's, when large numbers of insects were first recorded in the atmospheric convective layer (Glick 1939), researchers held the view that aerial dispersal was entirely dependent on weather conditions (e.g. passive dispersal; McManus 1988). Most empirical data since then suggested that there is a behavioural component to dispersal. For example, using radars to quantify airflow and movement of small insects in the atmosphere, Wainwright et al. (2017) detected movement velocities independent of airflow. Work on flies is consistent with the findings of Wainwright et al. (2017).

Desert species of Drosophila (D. mimica, D. nigrospiracula and D. mojavensis) disperse both up and down wind and this behaviour is dependent on food availability (Richardson and Johnston 1975; Markow and Castrezana 2000). In a recapture experiment, Coyne et al. (1982) released groups of $D$. melanogaster and its sister species D. simulans in the Death Valley and were able to recapture a proportion of them at an oasis several miles from the release site even though the flies faced cross winds during their flight. Although none of the studies on Drosophila explicitly tested wind effects on dispersal the results suggest that, for these flies, dispersal is not entirely passive. As is the case for the 
150 effects of temperature on dispersal, it is unknown whether individual flies respond similarly to wind

151 effects or whether there is a behavioural predisposition to respond differently to wind speed and

152 direction.

154 Here we examined the effects of temperature, and wind direction and speed on the dispersal behaviour

155 of rover and sitter D. melanogaster as well as on an outbred population of flies released in the field

156 using a mark/release/recapture experiment. Because flies do not exhibit dispersal behaviour in a strict

157 sense (e.g. departure, settlement), but rather move while foraging, searching for mates, avoiding

158 predators etc. we defined any movement away from a release site as dispersal. This idea fits well with

159 dispersal defined in its simplest form as any movement by individuals leading to spatial spread with

160 potential for genetic mixing (Ronce 2007). We quantify dispersal as the rate at which this spread occurs

161 (see analysis below). To investigate how the dispersal behaviour of rover, sitter and the outbred strain

162 of flies respond to temperature and wind we monitored the weather during the experiment. We

163 explicitly evaluated the general prediction that dispersal increases with temperature and whether our

164 more (rover) and less (sitter) dispersive strains differed from our outbred population. We also explicitly

165 tested whether flies use wind to disperse and we used a novel approach to detect the tendency for flies

166 to disperse either up or down wind in the field. We also determined whether the strains differed in their

167 dispersal in response to wind, both in terms of direction and velocity. Combining individual predictors

168 of dispersal with temperature and wind allowed us to evaluate not only the interaction between

169 individual dispersal propensity and the environmental factors, but also the relative contribution of each

170 factor in population-level dispersal in nature.

172 2. Materials and methods 
2.1 Fly lines

175 To evaluate how strain-differences in behaviour influence dispersal of flies in the field we used two

176 inbred strains (rover- for $^{\mathrm{R}}$ and sitter- $f \circ r^{\mathrm{s}}$ ) and one outbred strain of flies. The effect of for on the

177 dispersal strategy of the rover and sitter inbred strains was documented in Edelsparre et al. (2014) and

178 Edelsparre et al. (2018). The outbred population was established from 92 iso-female lines originally collected in Sudbury, Ontario, Canada (501484.97 E, 5143198.65 N UTM) on August 12, 2012 by

180 Thomas Merritt. Several months prior to the commencement of the field experiment each of the 92

181 lines were randomly assigned to one of $6170 \mathrm{~mL}$ sponge-topped plastic Drosophila bottles (4 bottles

182 each containing 15 different lines and 2 bottles each containing 16 lines) containing $40 \mathrm{~mL}$ of media as

183 described below and maintained as stocks. Two months prior to the field experiment the six bottles

184 were transferred to population cages and flies were allowed to mix for one generation in 16 open

185 bottles inside the population cage. Hereafter the 16 bottles were extracted from the cage, and brooded over for two more generations before a fresh generation of flies was used in the field experiment.

\subsection{Experimental field}

To quantify the movement of flies in the field, we prepared a $100 \mathrm{~m}$ by $100 \mathrm{~m}$ large experimental arena in an open meadow at the rare Charitable Research Reserve west of Cambridge, Ontario, Canada (553195.00 E, 4802841.00 N UTM) that was recently converted from agricultural land to a nature reserve (Fig. 1a). The field is flat with a sloping gradient of zero from corner to corner and mainly consists of vegetation such as goldenrods (Solidago spp.), asters (Symphyotrichum spp.), thistles

194 (Circium spp), and common milkweed (Asclepias syriaca). Vegetation reached an average height of observation). 
198 The experimental design was constructed with sampling points positioned in a gradient radiating away

199 from a central site from which the flies were released. The arrangement of each point was determined

200 by first specifying a $21 \times 21$ matrix with 441 points (corresponding to $100 \times 100$ metre square with

201 each point $5 \mathrm{~m}$ apart) and then by selecting specific points at which sampling locations were to be

202 placed. Locations were selected by first converting Cartesian coordinates to polar coordinates using a

203 trigonometric function that varied sampling concentration according to a cosine function and truncating

204 the continuous values such that positive values represented sampling locations and negative values

205 represented locations without sampling. This resulted in a total of 227 locations (including the central

206 release location) that varied in distance and density in a parameterized gradient (Fig. 1b). Arranging

207 sampling locations in this manner was done to balance the spatial resolution with the amount of time

208 required to sample the entire field (i.e. temporal resolution).

209

210 The experimental arena was prepared by first positioning each of the four corners $100 \mathrm{~m}$ apart in the

211 field and then building trap lines within the arena. The arena was $100 \times 100 \mathrm{~m}^{2}$ with 21 rows and 21

212 columns. Trap lines were positioned every $5 \mathrm{~m}$. Stake flags $(6.35 \times 8.89 \mathrm{~cm}$ on a $53 \mathrm{~cm}$ wire stem,

213 Milwaukee Tools) were placed at $5 \mathrm{~m}$ intervals along each trap line resulting in 441 flagged locations.

214 The field diagram (Fig. 1b) was used to identify flags where a given sampling location was to be

215 placed. One of the four corners of the arena was angled towards a North-Easterly direction. This was

216 due to the prevailing winds mainly moving in an East and East-North-East direction as measured by

217 our weather station (Vantage Vue, Davis Instruments, California, USA). For the same reason, the

218 release site at coordinate $(0,0)$ was off-set from the middle to allow higher sampling resolution in the

219 direction of the prevailing winds. The weather station was installed along the diagonal (Fig. 1c) to

220 measure wind direction and speed and temperature every 15 minutes. 
222 Sampling locations consisted of baited traps inserted into the ground (10 cm off ground). Traps were 223 prepared from a $90 \mathrm{~mL}$ plastic cup (Starplex, Starplex Scientific, Toronto, ON, Canada) positioned 224 horizontally on a small piece of bamboo (Figure 1d). Prior to inserting a trap, the sampling location was prepared by manually removing vegetation around the flag and placing a small piece of landscaping fabric (The Scotts Company LLC) on the ground surface. To prevent other insects such as ants from gaining access to a trap, the small piece of bamboo that held the trap was inserted through the inner part of a two-part plastic shot glass (81.3 mL Amscan, Toronto, ON, Canada). The outer part of the glass was filled with water, which served as a moat and effectively prevented access to the piece of bamboo holding the baited trap (Fig. 1d).

Each trap was baited with $20 \mathrm{~mL}$ of fly medium consisting of a mixture of sugar, dead yeast and agar.

Briefly, for approximately $1 \mathrm{~L}$ of medium we mixed $100 \mathrm{~g}$ of sugar, of which $50 \%$ of the sugar came

from sucrose and the other $50 \%$ came from bananas ( $0.12 \mathrm{~g}$ of sucrose in $1 \mathrm{~g}$ of banana) that were

blended prior to mixing, with $110 \mathrm{~g}$ of yeast and $17.43 \mathrm{~g}$ of agar in $1 \mathrm{~L}$ of tap water. We also added $8 \mathrm{~g}$

of $\mathrm{C}_{4} \mathrm{H}_{4} \mathrm{KNaO}_{6}, 1 \mathrm{~g}$ of $\mathrm{KH}_{2} \mathrm{PO}_{4}$ and $0.5 \mathrm{~g}$ each of $\mathrm{NaCl}, \mathrm{MgCl}_{2}, \mathrm{CaCl}_{2}$ and $\mathrm{Fe}_{2}\left(\mathrm{SO}_{4}\right)_{3}$ which was part of

the standard yeast-sugar-agar laboratory medium that we used for maintaining fly stocks (Belay et al.

2007). All compounds were combined, mixed for an hour and autoclaved to ensure that the medium were not biased towards any strain or sex used in the present study (results not shown). 
247 regression equation for each strain. Each equation was used to predict the number of flies in each vial 248 given their group weight and strain ID. Batches of approximately 150 flies were anesthetized with $\mathrm{CO}_{2}$ 249 and transferred to separate empty plastic vials after which a minute amount of dry fluorescent pigment 250 (DayGlo, Cleveland, OH, USA) was added (rover, Saturn Yellow, AX-17-N; sitter, Aurora Pink, AX251 5-11; outbred flies, Horizon Blue, A-19). The colours were assigned randomly to each strain and were 252 not known to the experimenters. Each vial was gently shaken to ensure that all flies were rolled in the 253 pigment and subsequently transferred to $170 \mathrm{~mL}$ sponge-topped plastic Drosophila bottles where they 254 were allowed 24 hours to groom themselves. This method left a badge on the ventral and dorsal 255 thoraces that can be visualized by a portable black light in the field. In total 5644 rovers, 5352 sitters 256 and 5657 outbred flies (2-7 days post eclosion) were tagged and released at the centre of the experimental field.

\subsection{Releasing and recapturing flies}

260 Prior to release, flies were acclimatized to their surroundings by placing the Drosophila bottles next to 261 the central release trap at 09:00 on 10 October 2015. At 12:00 flies were released by gently removing 262 the sponge-tops from each bottle in a haphazardly chosen order. It took 8 minutes from the release of 263 the first bottle to the release of the last bottle.

Recapturing flies involved first randomly choosing between rows and columns of the trapping arena

(Fig. 1b). Once either rows or columns were selected, three field observers sampled rows or columns in a random order. A complete sampling round involved visiting all 227 baited traps and bringing traps containing captured flies to a central location where all the samples were processed. Processing involved transferring flies to transparent Drosophila vials, identifying the colour markings by exposing flies to a black light and visually counting the number of flies with each colour at each sample location. 
capture location. Traps were baited with fresh food every second day. The first sampling round

commenced 30 minutes after release (12:30), and we strove to complete a minimum of two sampling

rounds per day over a course of five days (or until no or few flies remained on the experimental field).

A sampling round took a maximum of two hours depending on how many flies were captured. In total

12 sampling rounds were completed over the 5 days, however, because of low capture rates after 50

277

hours post-release we used the first six sampling rounds in our analysis corresponding to $0.5,1.5,21$,

26, 46 and 50 hours after the release (Fig. 2).

\subsection{Data analysis}

281 To investigate how the rover, sitter and outbred strains respond to temperature and wind in the field, we 282 developed and fit a dynamic spatio-temporal model to the data shown in Fig. 2. Dynamic spatio283 temporal modelling enables mathematical models, like partial differential equations, to be fit to data

For each fly-strain, we fit a partial differential equation that included a component that describes

In Eq. 1, $u(\mathbf{s}, t)$ is the likelihood an individual is at location $\mathbf{s} \equiv\left(s_{1}, s_{2}\right)^{\prime}$ at time $t$, which can be converted to the intensity of the dispersing population by multiplying $\theta \times u(\mathbf{s}, t)$, where $\theta$ is the 
296 For the analysis, we specified the diffusion rate as follows

$$
\mu(\mathbf{s}, t)=\left\{\begin{array}{cc}
e^{\alpha_{0}+\alpha_{1} z(\mathbf{s}, t)} & x(t)=1 \\
0 & x(t)=0
\end{array}, \quad \text { Eq. } 2\right.
$$

where $\alpha_{0}$ is the intercept and $\alpha_{1}$ is a regression coefficient for standardized temperature, $z(\mathbf{s}, t)$, at

location $\mathbf{s}$ and time $t$. The indicator variable $x(t)$ depends on the time, $t$, and is equal to 1 if it is

Similarly, we specified the advection rate as

$$
v(\mathbf{s}, t)=\left\{\begin{array}{cc}
\beta_{1} w(\mathbf{s}, t) & x(t)=1 \\
0 & x(t)=0
\end{array}, \quad \text { Eq. } 3\right.
$$

where $\beta_{1}$ is a regression coefficient for wind velocity, $w(\mathbf{s}, t)$, at location $\mathbf{s}$ and time $t$. During daylight, the advection rate, $v(\mathbf{s}, t)$, can be positive or negative. Thus, there is no constraint required

312 like the exponential function in Eq. 2. If the wind transports (advects) individuals, we expect a positive advection rate, whereas if individuals actively move into the wind then we expect a negative advection rate. Unlike the diffusion rate, the advection rate does not contain an intercept term because when the wind velocity is zero (i.e., $w(\mathbf{s}, t)=0)$ ), there should be no advection. Similar to temperature, wind 
By fitting Eq. 1 to the data for each strain, we are able to estimate parameters that describe each strain's

movement in response to changes in the environment. For example, the magnitude and sign of the

estimated value of $\alpha_{1}$ allows us to infer how the diffusion rate for each strain changes when the

temperature increases. We provide a brief description below of how we fit partial differential equations

to data. A more detailed description is provided in supporting material along with computer code (see

Fitting Eq. 1 to the data from each strain first involves specifying a statistical model for the observed

data. We assumed

$$
y_{i}\left(\mathbf{s}_{j}, t\right) \sim \text { negative binomial }\left(\lambda_{i}\left(\mathbf{s}_{j}, t\right), \phi\right), \quad \text { Eq. } 4
$$

where $y\left(\mathbf{s}_{j}, t\right)$ is the number of individual of strain $i(i=1,2,3)$ captured at the location $\mathbf{s}_{j}$

corresponding to the trap locations (i.e., $j=1,2, \ldots, 227$ ) at the time $t$. For our data, the observed times

modeled with

$$
\lambda_{i}\left(\mathbf{s}_{j}, t\right)=\left\{\begin{array}{c}
\mathrm{p}_{i} \theta_{i} u_{i}\left(\mathbf{s}_{j}, t\right), \quad \text { with proability } \psi_{i} \\
\mathrm{p}_{i} \theta_{i} \frac{1}{|\mathcal{S}|}, \quad \text { with probability } 1-\psi_{i}
\end{array} \quad \text { Eq. } 5\right.
$$

In Eq. 5, $\mathrm{p}_{i}$ is the capture probability, $\theta_{i} u_{i}\left(\mathbf{s}_{j}, t\right)$ is the intensity of the dispersing population from the

Eq. 1 (where $\theta_{i}$ is the known number of individuals of strain $i$ released), $\frac{1}{|\mathcal{S}|}$ is a uniform probability 
341 the uniform distribution exhibit abnormal behaviour and could be caught with equal chances at any

342 location within the study area. For example, upon release a small proportion of the flies exhibited

343 bolting behaviour. Because these behaviours are abnormal, we expected that the estimated value of the 344 parameter $\psi_{i}$ would be close to one.

346 We took a Bayesian approach for parameter estimation and specified priors for all unknown

347 parameters. To estimate the parameters in our model from the data, we developed software using a

348 Markov chain Monte Carlo algorithm similar to the approach described in Hooten and Hefley (2019,

349 Ch 28 pg. 501); however, solving Eq. 1 involved developing an emulator described by Hooten et al.

350 (2011). Details of the implementation are provided in Appendix S1.

3. Results

In total, we captured 1223 rovers, 861 sitters and 279 outbred flies over the five days of the experiment.

Approximately 95\% (2363 flies) of all captured flies were captured within the first 50 hours of the

experiment, corresponding to the first six sampling rounds. The capture data from the six sampling

rounds used in the analysis are described in Figure 2. During the first 50 hours from release, the flies

experienced a range of changing weather conditions, ranging from $10-22{ }^{\circ} \mathrm{C}$ during the day and nightly

temperatures ranging from just above $5{ }^{\circ} \mathrm{C}$ to $16{ }^{\circ} \mathrm{C}$ (Fig. 3a). Wind speeds ranged from 0 to just above

East-North-East and East-South-East directions (Fig. 3c).

363 In general, our prediction that dispersal increased with temperature was supported. This conclusion is

364 based on the finding that the posterior mean of the diffusion rates for rovers and sitters increased

365 rapidly from $18{ }^{\circ} \mathrm{C}$ to $22{ }^{\circ} \mathrm{C}$ (Fig. 4). The posterior distribution of the diffusion rates show that dispersal 
of both sitters and rovers responded positively to temperature, but the magnitude of the response is

367 uncertain, particularly at temperatures above $20^{\circ} \mathrm{C}$. In addition, the posterior mean estimates for rovers

368 and sitters mirrored each other particularly at temperatures below $18{ }^{\circ} \mathrm{C}$, but tended to be greater than

369 the diffusion rate for outbred flies, which largely remained unchanged across the temperature range

370 (Fig. 4). For example, if the temperature was $22^{\circ} \mathrm{C}$ over a course of 24 hours, our results indicate that

371 we should expect rovers and sitters to spread $79 \mathrm{~m}^{2}$ and $152 \mathrm{~m}^{2}$ more when compared to the outbred

372 strain. Conversely, at temperatures below $14{ }^{\circ} \mathrm{C}$ the outbred strain spreads at least $36 \mathrm{~m}^{2}$ more over 24

373 hours when compared to rovers and sitters. The posterior distribution for all parameter estimates for the

374 temperature analysis are shown in Fig. 6a and 6b.

375

376 Wind speed and direction played a significant role in dispersal for all three fly strains. This conclusion

377 is supported by the finding that the advection parameter was positive and increased with wind speed for

378 rovers, sitters and outbred flies (Fig. 5). In fact, even with the level of uncertainty around each posterior

379 mean the lower limit of the $95 \%$ credible interval for all three strains remains above zero even at wind

380 speeds below $0.5 \mathrm{~m} / \mathrm{s}$. Although there is uncertainty in the estimates (cf. $95 \%$ credible intervals on Fig.

381 5), the difference among posterior mean responses becomes larger as wind speed increases. For

382 example, sitters were most sensitive to wind speeds whereas rovers were the least sensitive; at a wind

383 velocity of $1 \mathrm{~m} / \mathrm{s}$ sitters are expected to advect approximately 5 metres further over 24 hours than

384 rovers, however, at wind velocities of $2.5 \mathrm{~m} / \mathrm{s}$ the difference between the two strains is expected to be

385 approximately 13 metres over 24 hours. The parameter estimates for the advection part of the analysis

386 are shown in Fig. 6c. 
390 A number of conclusions can be drawn from the results of our study. First, temperature plays a critical

391 role in fly dispersal in the field. We found that for rovers and sitters, the posterior mean of the diffusion 392 rates increased with temperature particularly between 18 and $22{ }^{\circ} \mathrm{C}$. In contrast, the posterior mean of 393 the diffusion rate of the outbred fly strain tended to be greater than the diffusion rates of rovers and 394 sitters at temperatures less than $18{ }^{\circ} \mathrm{C}$, but largely remained unchanged across the temperature range. 395 The rover and sitter responses to temperature mirror each other. However, the effect size of the mean 396 estimates particularly between the rover and the outbred strain is noteworthy. For example, at $22{ }^{\circ} \mathrm{C}$ the 397 mean diffusion rate is nearly four times larger for rovers than for the outbred strain (Fig. 4), suggesting 398 that the models have the capacity to predict strain-dependent differences in dispersal outcomes over 399 time. Second, wind plays a critical role in the dispersal of flies. The advection rate increased linearly 400 with wind speed for all strains, however, rovers tended to be less sensitive to the effect of wind speed 401 relative to sitters and outbred flies. As was the case for temperature, there is a relatively large amount 402 of uncertainty in the magnitude of the response of sitters, rovers and outbred flies to wind speed, as 403 indicated by the $95 \%$ credible intervals, however, there is strong evidence that all three strains 404 responded positively to increasing wind speed. In addition, the posterior mean advection distance 405 between rovers and sitters more than doubled for every $1 \mathrm{~m} / \mathrm{s}$ increase in wind speed (Fig. 5). This 406 suggests that flies were influenced by both the direction and the speed of wind during the experiment, 407 but that the magnitude of this effect tended to depend on fly-strain. This suggests that differences in 408 down wind dispersal were due to behavioural differences and not a passive response. Thirdly, 409 combining ecological diffusion modelling with wind advection clearly improved our ability to predict 410 dispersal at the population-level. This conclusion is based on the finding that dispersal of flies in the 411 field varied with both temperature and wind. For temperature, the posterior means of the diffusion rates 412 increased rapidly for rovers and sitters, while the rate remained unchanged for the outbred population. 413 For wind the effect is even stronger. The posterior means of the advection rates were above zero across 414 the range of wind speeds for all three strains. Even with the level of uncertainty in the estimates, the 
415 lower limit of the $95 \%$ credible intervals for all three posterior means is above zero at low wind

416 speeds. This further strengthens the evidence in favour of a strong wind effect. Although there was

417 some evidence of differences in response to wind speed, overall rovers, sitters and the outbred

418 population exhibited similar patterns of response to wind (parallel responses). Taken together the

419 environmental effects were strong for both the rover and sitter strains as well as the outbred population,

420 although the strength of the evidence varied across the temperature and wind ranges. Our models

421 therefore captured the effects of key descriptors of fly movement that a model without temperature and

422 wind likely would have ignored and therefore our results inform our understanding of potential factors

423 underlying dispersal in nature.

Climate driven environmental conditions such as temperature and wind have long been considered key

factors influencing fly dispersal in particular and insect dispersal in general (Glick 1941, McManus

1988). Since the 1950's prognoses of the relationship between temperature, wind, and dispersal have

been used to both forecast and backtrack incidents of migrant pests such as African armyworms and

desert locusts (Wellington 1954, Rainey 1979). In support of this, we found that wind speed and

direction played a significant role in dispersal even at extremely low wind speeds. This suggests that

wind transport can underlie a large part of the estimated rate of dispersal. We detected wind speeds

between 0 and $3 \mathrm{~m} / \mathrm{s}$ during this study. As such, wind may play an even larger role when speeds exceed

$3 \mathrm{~m} / \mathrm{s}$. Clearly, in our study flies were able to navigate towards, around and into the traps. Taylor

(1974) used the term "boundary layer" to describe a hypothetical layer of air near the ground where

wind is not able to affect the movement of small insects (i.e. insect flight speed exceeds wind speed). 
cannot be directly answered by our data. Nevertheless, the low wind speeds and moderate to warm

temperatures experienced during our study were conducive to insect movement (Glick 1942, Cockbain

1961, Taylor 1963).

443 Our findings demonstrate the potential use of genetic and environmental information in detecting 444 individuals that may influence dispersal disproportionally. Although we did not address genetic effects 445 directly in this study, we used different strains of flies, rovers (more dispersive) and sitters (less 446 dispersive) and an outbred strain, to understand how flies with different genetic predispositions to 447 disperse influenced the spatial spread of a fly-population. Unlike previous findings in the laboratory 448 and the field (Edelsparre et al. 2014, Edelsparre et al. 2018), the known difference in dispersal between 449 rovers and sitters was minimized in response to temperature and wind conditions. Sitters tended to 450 disperse faster with wind than did rovers particularly at high wind speeds. This suggests that an understanding of dispersal likely hinges on disentangling how genes involved in dispersal interact with relevant factors in the environment to affect individual differences in dispersal behaviour (Sokolowski 2001, Dudaniec et al. 2018, Saastamoinen et al. 2018). Insect movement involves multiple genes (Saastamoinen et al. 2018) in interaction with many environmental factors including temperature and wind measured in the present study. for is one of the genes that affects movement related behaviours in a wide range of insect species, including ants (Ingram et al. 2005) and honey bees (Ben-Shahar et al. 2002). for has also recently been associated with outbreaks of locusts (Tobbak et al. 2013) and possibly spruce budworm, Choristoneura fumiferana (Van Hezewijk et al. 2018). Describing the role of for and other candidate genes in invasive species should provide a unique opportunity to better understand and model invasion biology in terrestrial environments. Additionally, by extending this thinking to include temperature and wind our findings are particularly pertinent to elucidating conditions under which 
463 dispersal offer the potential to reduce uncertainty in invasion biology where unpredictability seems to

464 be the rule rather than the exception (Melbourne and Hastings 2009).

465

466 Our findings provide a powerful framework for combining individual-level predictors with climate 467 driven variables to understand dispersal at the population-level. An implicit assumption in many 468 models, including diffusion models, is that the dispersing individuals within the population are 469 identical (Kot et al. 1996, Gurarrie et al. 2009). Other studies have proposed individual differences in 470 behaviour as an explanation for non-random variation in dispersal frequently reported in the literature 471 (Skalski and Gilliam 2000, Fraser et al. 2001, Réale et al. 2007). We addressed this assumption directly 472 by using the rover, sitter and outbred strains of flies as proxies for individual differences in dispersal 473 propensity. Through the fitting of separate models for each strain we show how such efforts can be 474 useful predictors of population-level dispersal in the field. Our results are consistent with models that 475 propose individual variation in behaviour as explanations for population-level variation in dispersal.

476 Our findings not only provide the potential to inform how individuals influence dispersal 477 disproportionally but they also improve our understanding of key mechanisms surrounding individual 478 differences in response to environmental factors that give rise to population-level dispersal.

There are potential limitations to our study. First, the temporal resolution we used in our experimental design affected the power of our analysis. Increasing temporal sampling over spatial sampling could have provided stronger evidence for differences among strains. One way to increase the temporal resolution may be to sample flies as presence/absence data rather than abundance. This would reduce the time spent at each sampling location and increase the number of times the entire arena could be surveyed in a day. Second, the rover and sitter strains have been cultured in the laboratory for over 30 years and may not have recapitulated dispersal behaviour in natural populations. The dispersal rate of 
reported in the literature (Cockbain 1961, Taylor 1963). Along with the rover and sitter strains, we

released an outbred strain established from the field. This strain was assembled in 2015 using 92

isofemale lines collected in 2012. The diffusion rate of this outbred strain was stable across the

temperature range in comparison to the rover and sitter strains. The outbred strain was collected near

the limit of D. melanogaster's northern-most distribution in Sudbury Ontario, Canada (Thomas Merritt,

personal communication). This might explain why the outbred strain tended to be more active at low

temperatures than the rover and sitter strains that were originally caught well within $D$. melanogaster's

normal range (Toronto, Ontario, Canada, $402 \mathrm{~km}$ south of Sudbury). Overall, our results provide a

useful approach for investigating how behavioural differences might lead to dispersal outcomes for a

population with multiple dispersal strategies and how those strategies may interact with temperature

and wind.

Our study provides two scientific contributions to our understanding of the evolution and ecology of

organismal movement. First, we took advantage of the wealth of information available for flies in

general and this Drosophila melanogaster model system in particular to build models that incorporated

and climatic effects. Earlier attempts to understand the movement of organisms generally relied on 
513 better understand the distribution of organisms in nature. Second, we developed a powerful framework

514 for linking individual variation in dispersal strategy with the environment. Understanding how such

515 components can lead to population-level dispersal is valuable because individual differences in

516 behaviour are increasingly linked with important ecological and evolutionary processes such as habitat

517 fragmentation (Cote et al. 2017), climate change (Fitzpatrick and Edelsparre 2018), and biogeography

518 (Canestrelli et al. 2016). In fact, individual variation in behaviour has recently been referred to as a

519 'pacemaker' of evolution of non-behavioural traits. Our study therefore offers a unique opportunity to

520 understand how dispersal strategy influences the distribution of populations and ultimately the pattern

521 of biological diversity on our planet (Canestrelli et al. 2016).

522

523 Acknowledgements

525 We acknowledge that this study was carried out on traditional and sovereign territory of the

526 Onkwehonwe Peoples of Six Nations of the Grand River and the Anishinaabe Peoples of the

527 Mississaugas of the New Credit and is within the territory of the Neutral Peoples. This study was

528 possible because of the historical and ongoing indigenous stewardship of these lands and remain

529 critical to biodiversity in Ontario. We also thank rare Charitable Research Reserve, Cambridge,

530 Ontario for facilitating our use of the field site, providing field equipment as well as providing access to

531 field laboratory and accommodation during our stay at the reserve. We would particularly like to thank

532 Jenna Quinn the Program Scientist at rare for making our stay at rare smooth and enjoyable. We are

533 deeply grateful for the assistance of Anders Vesterberg and Jenna Chen whose clever and meticulous

534 efforts helped setting up and executing the experiment as well as collecting the data. Lastly, we are

535 grateful to Thomas Merritt for collecting the iso-female lines that produced the outbred population.

536 This research was funded by Natural Sciences and Engineering Research Council (NSERC) to MJF, 
537 MAR and MBS. Partial funding for this project was provided by the National Science Foundation 538 (NSF) via grant DEB 1754491 to TJH.

539

540 References

541 Anreiter, I. and M. B. Sokolowski. 2019. The foraging gene and its behavioral effects: pleiotropy and 542 plasticity. Annual Review Genetics. 53: 373-392.

544 Belay, A. T., R. Scheiner, A. K. -C. So, S. J. Douglas, M. Chakaborty-Chatterjee, J. D. Levine and M.

545 B. Sokolowski. 2007. The foraging gene of Drosophila melanogaster: spatial-expression analysis and 546 sucrose responsiveness. Journal of Comparative Neurology. 504: 570-582.

Ben-Shahar Y., A. Robichon, M. B. Sokolowski, and G. E. Robinson. 2002. Influence of gene action across different time scales on behavior. Science. 296: 741-744.

Brisson, J. A. 2010. Aphid wing dimorphisms: linking environmental and genetic control of trait variation. Philosophical Transactions of the Royal Society B. 365: 605-616.

Bocxlaer, I. V., S. P. Loader, K. Roelants, M. Menegon and F. Bossuyt. 2010. Gradual adaptation toward a range-expansion phenotype initiated the global radiation of toads. Science. 327: 679-682.

557 Canetrelli, D., R. Bisconti and C. Carere. 2016. Bolder takes all? The behavioral dimension of 
560 Clarke, J. S., S R. Carpenter, M. Barber, S. Collons, A. Dobson, J. A. Foley, D. M. Lodge, M. Pascual,

561 R. Pielke Jr., W. Pizer, C. Pringle, W. V. Ried, K. A. Rose, O. Sala, W. H. Schlesinger, D. H. Wall and 562 D. Wear. 2001. Ecological forecasts: an emerging imperative. Science. 293: 657-660.

563

564 Cote, J. and J. Clobert. 2007. Social personalities influence natal dispersal in a lizard. Proceedings of 565 the Royal Society B. 274: 383-390.

566

567 Cote, J., E. Bestion, S. Jacob, J. Travis, D. Legrand and M. Baguette. 2017. Evolution of dispersal 568 syndromes in fragmented landscapes. Ecography. 40: 56-73.

569

570 Cockbain, A. J. 1961. Low temperature thresholds for flight in Aphis fabae Scop. Entomologia $571 \quad$ Experimentalis et Applicata. 4: 211-219.

572

573 Coyne, J. A., I. A. Boussy, T. Prout, S. H. Bryant, J. S. Jones and J. A. Moore. 1982. Long-distance 574 migration of Drosophila. The American Naturalist. 119: 589-595.

575

576 Denno, R. F., Roderick, G. K., Olmstead, K. L., \& Döbel, H. G. 1991. Density-related migration in 577 planthoppers (Homoptera: Delphacidae): the role of habitat persistence. The American Naturalist. 138: $578 \quad 1513-1541$.

580 Duckworth, R. A. and A. V. Badyaev. 2007. Coupling of dispersal and aggression facilitates the rapid 581 range expansion of a passerine bird. Proceedings of the National Academy of Sciences USA. 104: 15017-15022. 
584 Dudaniec, R. Y., C. J. Yong, L. T. Lancaster, E. I. Svensson, and B. Hansson. 2018. Signatures of local 585 adaptation along environmental gradients in a range-expanding damselfly (Ischnura elegans).

586 Molecular Ecology. 27: 2576-93.

587

588 Edelsparre, A. H., M. J. Fitzpatrick, M. A. Rodríguez and M. B. Sokolowski. 2020. Tracking dispersal

across a patchy landscape reveals a dynamic interaction between genotype and habitat structure. Oikos.

590

DOI: https://doi.org/10.1111/oik.07368.

591

592 Edelsparre, A.H., McLaughlin, R.L. \& Rodríguez, M.A. 2013. Risk taking not foraging behavior 593 predicts dispersal of recently emerged stream brook charr (Salvelinus fontinalis). Ecosphere. 4: 73. 594

595 Edelsparre, A. H., S. Shahid and M. J. Fitzpatrick. 2018. Habitat connectivity is determined by the 596 scale of habitat loss and dispersal strategy. Ecology and Evolution. 8: 5508-5514.

Edelsparre, A. H., A. Vesterberg, J. H. Lim and M. J. Fitzpatrick. 2014. Alleles underlying larval foraging behaviour influence adult dispersal in nature. Ecology Letters. 17: 333-339.

600

601 Fitzpatrick, M. J. and A. H. Edelsparre. 2018. The genomics of climate change. Science. 359: 29-30.

602 Freedman, L. A., S. Conant and R. C. Fleischer. 1987. Evolutionary ecology and radiation of Hawaiian 603 passerine birds. Trends in Ecology and Evolution. 2: 196-203.

604

605

Fraser, D. F., J. F. Gilliam, M. J. Daley, A. N. Le and G. T. Skalski. 2001. Explaining leptokurtic 
609 Garlick, M. J., J. A. Powell, M. B. Hooten and L. R. McFarlane. 2012. Homogenization of large-scale

610 movement models in ecology. Bulletin of Mathematical Biology. 73: 2088-2108.

611

612 Glick, P. A. 1939. The distribution of insects, spiders, and mites in the air. United States Department of 613 Agriculture Technical Bulletin. 673: 1-151.

614

615 Glick, P. A. 1942. Insect population and migration in the air. pp. 88-98 in Moulton, S. (Ed.),

616 Aerobiology. American Association of Advanced Science. Washington, D.C. A.A.A.S. Publ. 17.

617

618 Gomez-Uchida, D., D. Canas-Rojas, C. M. Riva-Rossi, J. E. Ciancio, M. A. Pascual, B. Ernst, E. Aedo,

619 S. S. Musleh, F. Valenzuela-Aguayo et al. 2018. Genetic signals of artificial and natural dispersal

620 linked to colonization of South America by non-native Chinook salmon (Oncorhynchus tshawytscha).

621 Ecology and Evolution. 00: 1-18.

622

623 Grant, P. R. 1981. Speciation and the adaptive radiation of Darwin's finches. American Scientist. 69:

$624 \quad 653-663$.

625

626 Gurarie, E., J. J. Anderson and R. W. Zabel. 2009. Continuous models of population-level

627 heterogeneity inform analysis of animal dispersal and migration. Ecology. 90: 2233-2242.

628

629 Hanski, I. and M. Gilpin. 1991. Metapopulation dynamics: brief history and conceptual domain.

630 Biological Journal of the Linnean Society. 42: 3-16.

631 
632 Hastings, A., K. Cuddington, K. F. Davies, C. J. Dugaw, S. Elmendorf, A. Freestone, S. Harrison, M.

633 Holland, J. Lambrinos et al. 2005. The spatial spread of invasions: new developments in theory and 634 evidence. Ecology Letters. 8: 91-101.

635

636 Hefley, T. J., M. B. Hooten, R. E. Russel, D. P. Walsh and J. A. Powell. 2017. When mechanism

637 matters: Bayesian forecasting using models of ecological diffusion. Ecology Letters. 20: 640-650.

Hilbe, J. M. (2011). Negative Binomial Regression. Cambridge University Press.

640

Hill, J. K., C. D. Thomas, D. S. Blakeley. 1999. Evolution of flight morphology in a butterfly that has

recently expanded its geographic range. Oekologia. 121: 165-170.

Hooten, M. B., M. J. Garlick, and J. A. Powell. 2013. Computationally efficient statistical differential equation modeling using homogenization. Journal of Agricultural, Biological, and Environmental

Statistics. 18: 405-428.

Hooten, M. B., Leeds, W. B., Fiechter, J., \& Wikle, C. K. (2011). Assessing first-order emulator

inference for physical parameters in nonlinear mechanistic models. Journal of Agricultural, Biological, and Environmental Statistics. 16: 475-494.

652 Hooten, M.B. and T.J. Hefley. 2019. Bringing Bayesian Models to Life. Chapman and Hall/CRC.

653 Ingram K. K., P. Oefner, D. M. Gordon. 2005. Task-specific expression of the foraging gene in har654 vester ants. Molecular Ecology. 14: 813-818. 
Johnson, C. G. 1969. Migration and Dispersal of Insects by Flight. Methuen \& Co Ltd. London. 763

657 pp.

658

659 Konopka, R. J. and S. Benzer. 1971. Clock mutants of Drosophila melanogaster. Proceedings of the

660 National Academy of Sciences of the United States of America. 68: 2112-2116.

661

662 Korsten, P., T. van Overveld, F. Adriaensen and E. Matthysen. 2013. Genetic integration of local 663 dispersal and exploratory behaviour in a wild bird. Nature Communications. 4: 2362.

664

665 Kot, M., M. A. Lewis and P. van den Driessche. 1996. Dispersal data and the spread of invading 666 organisms. Ecology. 77: 2027-2042.

667

668 Lombaert, E., A. Estoup, B. Facon, B. Joubart, J. C. Grégoire, A. Jannin, A. Blin, and T. Guillemaud. 669 2014. Rapid increase in dispersal during range expansion in the invasive ladybird Harmonia axyridis. 670 Journal of Evolutionary Biology. 27: 508-517. and determinism in replicated adaptive radiations of island lizards. Science. 279: 2115-2118.

675 Markow, T. A. and S. Castrezena. 2000. Dispersal in cactophilic Drosophila. Oikos. 89: 378-386.

676 Melbourne, B. A., and A. Hastings. 2009. Highly variable spread rates in replicated biological 677 invasions: fundamental limits to predictability. Science. 325: 1536-1539. 
McManus, M. L. 1988. Weather, behaviour and insect dispersal. Memoirs of the Entomological Society

680 of Canada. 146: 71-94.

681

682 Melbourne, B. A. and A. Hastings. 2009. Highly variable spread rates in replicated biological 683 invasions: fundamental limits to predictability. Science. 325: 1536-1539.

684

685 Myles-Gonzalez, E., G. Burness, S. Yavno, A. Rooke and M. G. Fox. 2015. To boldly go where no 686 goby has gone before: boldness, dispersal tendency, and metabolism at the invasion front. Behavioural 687 Ecology. 26: 1083-1090.

688

Nathan, R. 2008. An emerging movement ecology paradigm. Proceedings of the National Academy of

Sciences of the United States of America. 105: 19050-19051.

691

Nathan, R., W. M. Getz, E. Revilla, M. Holyoak, R. Kadmon, D. Saltz and P. Smouse. 2008. A

Academy of Sciences of the United States of America. 105: 19052-19059.

695

O’Riain, M. J., J. U. M. Jarvis and C. Faulkes. 1996. A dispersive morph in the naked mole rat. Nature.

380: 619-621.

698

Osborne, K. A., A Robichon, E. Burgess, S. Butland, R. A. Shaw, A. Coulthard, H. S. Pereira, R. J. protein kinase of Drosophila. Science. 227: 834-836. 
703 Phillips, B. L. 2009. The evolution of growth rates on an expanding range edge. Biology Letters. 5:

$704 \quad 802-804$.

705

706 Phillips, B. L., G. P. Brown, J. K. Webb and R. Shine. 2006. Invasion and the evolution of speed in 707 toads. Nature. 439: 803.

708

709 Rainey, R. C. 1979. Interactions between weather systems and population of locusts and noctuids in

710 Africa. pp. 109-119. In Rabb, R. L. and G. G. Kenedy (Eds.), Movement of highly mobile insects:

711 concepts and methodology in research. North Carolina State Univ. Raleigh. 455 pp.

712

713 Réale, D., S. M. Reader, D. Sol, P. T. McDougall and N. J. Dingemanse. 2007. Integrating animal

714 temperament with ecology and evolution. Biological Reviews. 82: 291-318.

715

716 Richardson, R. H. and J. S. Johnston. 1975. Behavioral components of dispersal in Drosophila mimica.

717 Oecologia. 20: 287-299.

718

719 Ronce, O. 2007. How does it feel to be like a rolling stone? Ten questions about dispersal evolution.

720 Annual Review of Ecology, Evolution and Systematics. 38: 231-253.

721

722 Saastamoinen, M., G. Bocedi, J. Cote, D. Legrand, F. Guillaume, C. W. Wheat, E. A. Fronhofer, C.

723 Garcia, R. Henry, A. Husby, et al. 2018. Geneticsof dispersal. Biological Reviews. 93: 574-599. 
725 Skalski, G. T. and J. F. Gilliam. 2000. Modeling diffusive spread in a heterogeneous population: a

726 movement study with stream fish. Ecology. 81: 1685-1700.

727

728 Skellam, J. G. 1951. Random dispersal in theoretical populations. Biometrika. 38: 196-218.

729

730 Sokolowski, M. B. 2001. Genetics meets behaviour. Nature Reviews Genetics. 2: 879-890.

731

732 Sokolowski, M. B., C. Kent and J. Wong. 1984. Drosophila larval foraging behaviour: developmental 733 stages. Animal Behaviour. 32: 645-651.

734

735 Taylor, L. R. 1963. Analysis of the effect of temperature on insects in flight. Journal of Animal 736 Ecology. 32: 99-117.

737

738 Taylor, L. R. 1974. Insect migration, flight periodicity, and the boundary layer. Journal of Animal 739 Ecology. 43: 225-238.

740

741 Tobback, J., H. Verlinden, K. Vuerinckx, R. Vleugels, J. Vanden Broeck \& R. Huybrechts. 2013.

742 Developmental-and food-dependent foraging transcript levels in the desert locust. Insect Science. 20:

743 679-688.

744

745 Van Hezewijk B., D. Vertman, D. Stewart, C. Béliveau, and C. Cusson. Environmental and genetic 746 influences on the dispersal propensity of spruce budworm (Choristoneura fumiferana). 2018.

747 Agricultural and Forest Entomology. 20: 433-441. 
749 Wainwright, C. E., P M. Stepanian, D. R. Reynolds and A. M. Reynolds. 2017. The movement of small

750 insects in the convective boundary layer: linking pattern with process. Scientific Reports. 7: 5438.

751

752 Wellington, W. G. 1954. Atmospheric circulation processes and insect ecology. The Canadian

753 Entomologist. 86: 312-333.

754

755 Wikle, C. K. 2003. Hierarchical Bayesian models for predicting the spread of ecological processes.

756 Ecology. 84: 1382-1394.

757

758 Wikle, C.K., A. Zammit-Mangion, and N. Cressie. 2019. Spatio-Temporal Statistics with R. Chapman 759 and Hall/CRC.

760

761 Zera, A. J. and R. F. Denno. 1997. Physiology and ecology of dispersal polymorphism in insects.

762 Annual Review of Entomology. 42: 207-230.

763

764

765

766

767

768

769

770 
bioRxiv preprint doi: https://doi.org/10.1101/2021.01.04.425125; this version posted January 5, 2021. The copyright holder for this preprint (which was not certified by peer review) is the author/funder, who has granted bioRxiv a license to display the preprint in perpetuity. It is made available under aCC-BY-NC 4.0 International license.

$771 \quad \underline{\text { Figures }}$

772

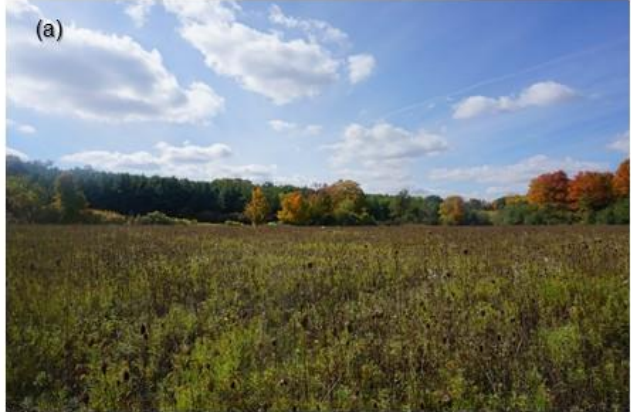

(c)

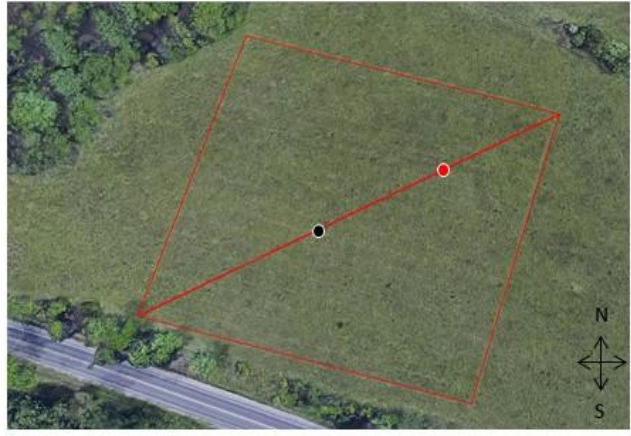

(b)

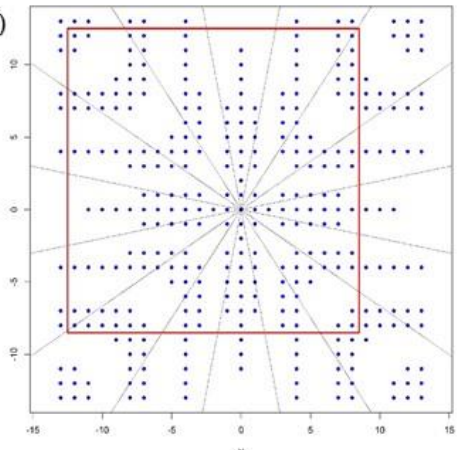

(d)

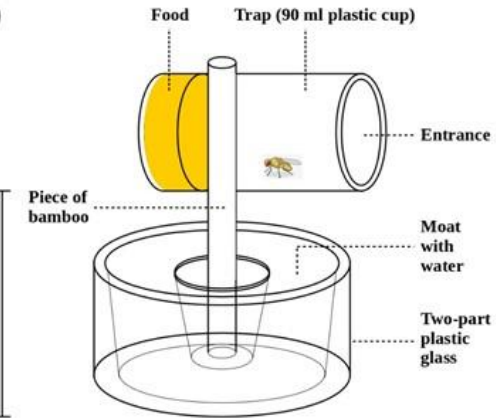

773

774 Figure 1.

775

776

777

778

779

780

781

782 
bioRxiv preprint doi: https://doi.org/10.1101/2021.01.04.425125; this version posted January 5, 2021. The copyright holder for this preprint (which was not certified by peer review) is the author/funder, who has granted bioRxiv a license to display the preprint in perpetuity. It is made available under aCC-BY-NC 4.0 International license.
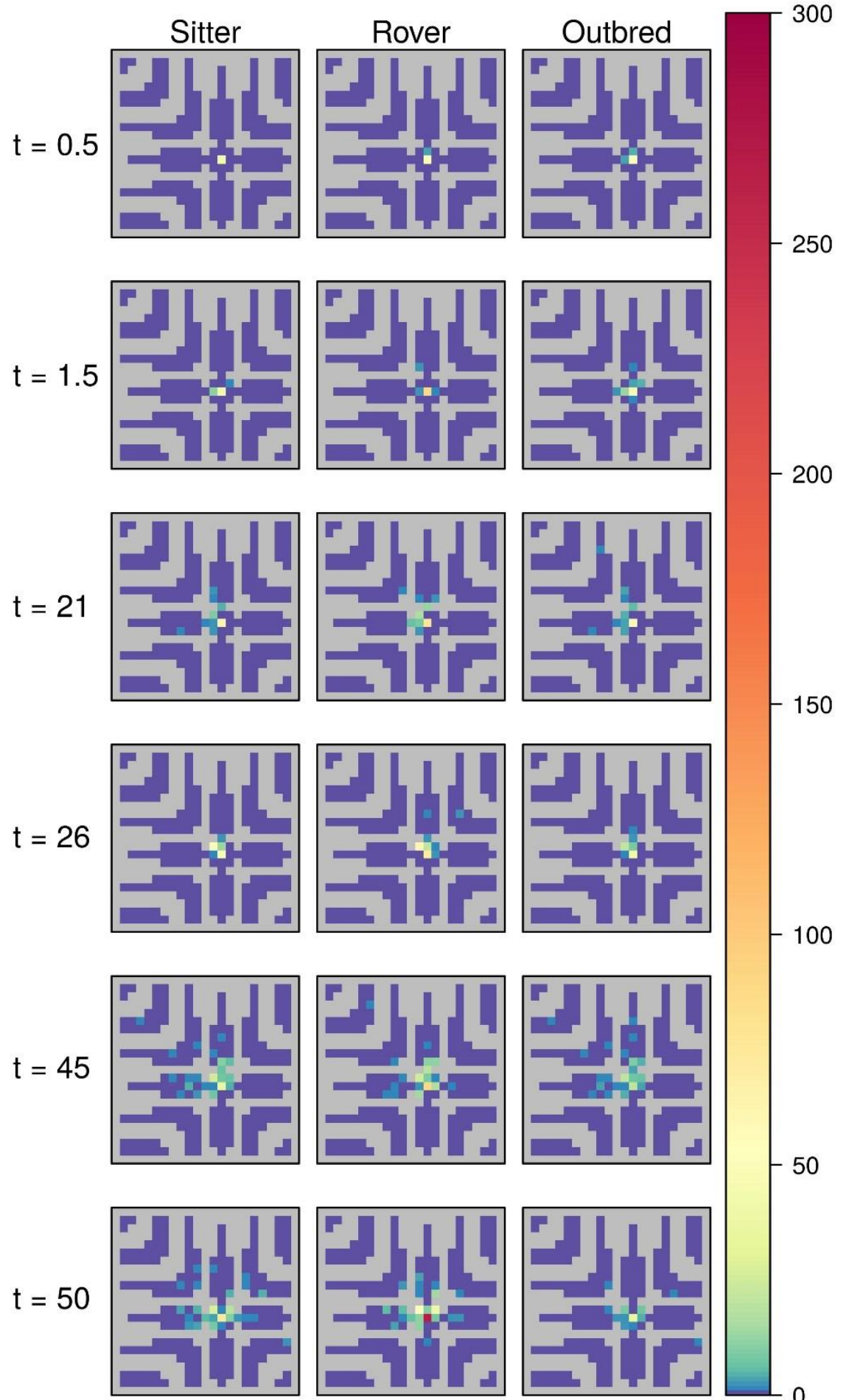

Figure 2. 
786

(a)

787

788

789

790

791

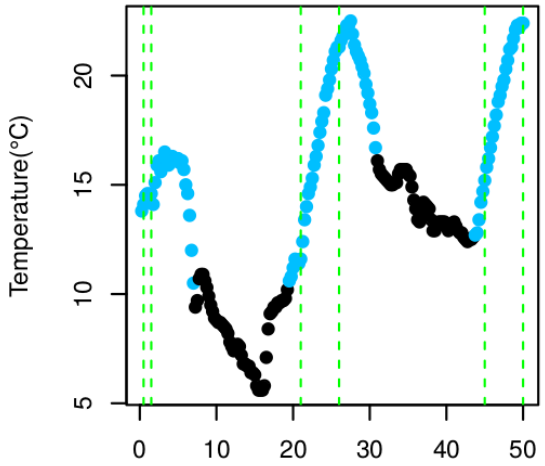

Time since release (hours) (b)

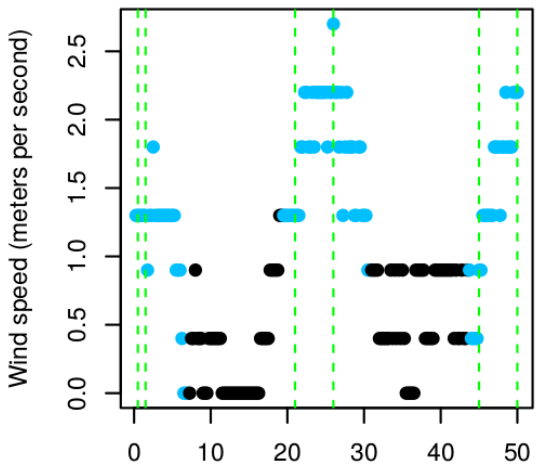

Time since release (hours) (c)

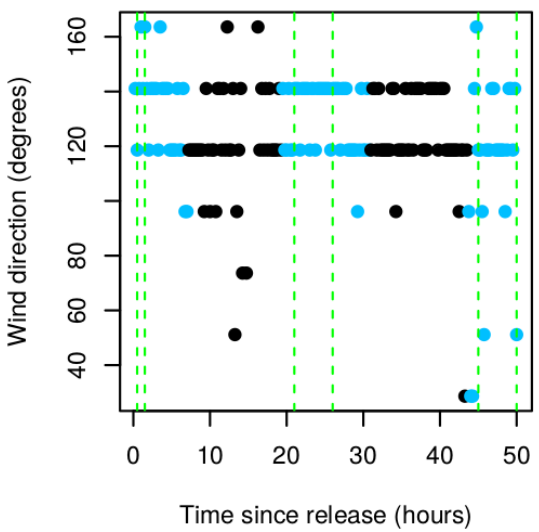

792 Figure 3.

793

794

795

796

797

798

799

800

801

802

803

804 


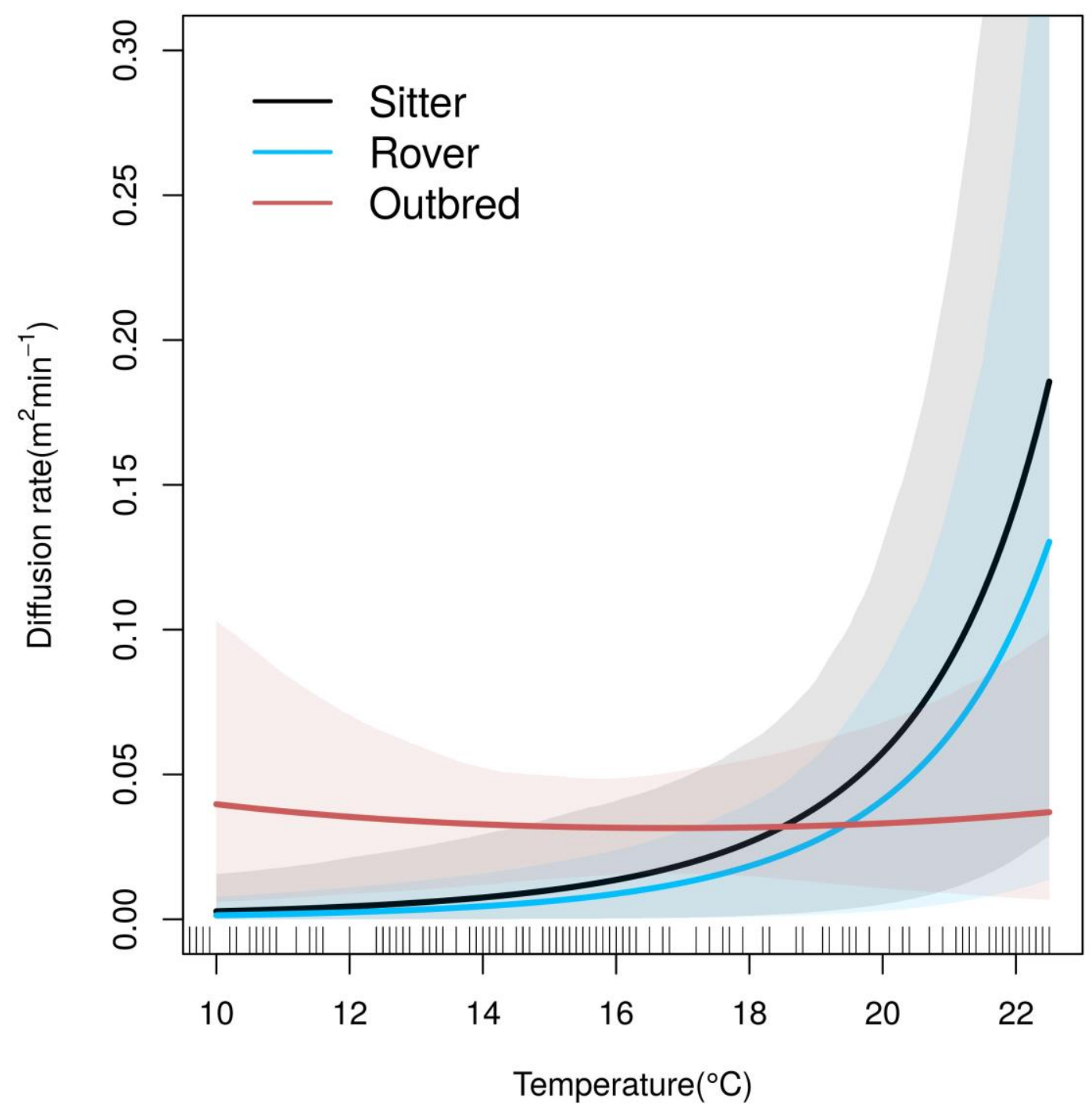

806 Figure 4

807

808 


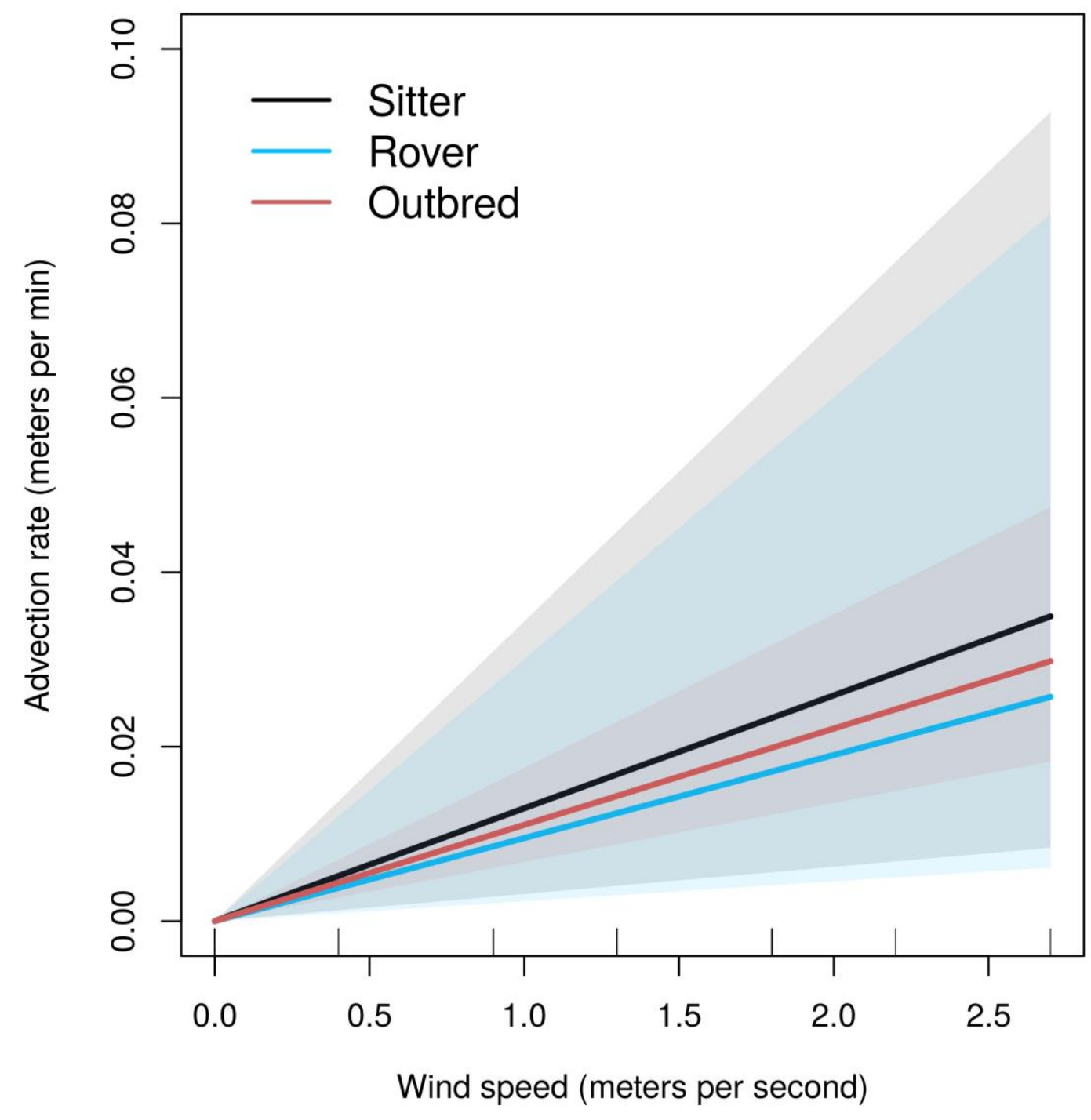

810 Figure 5.

811

812

813

814

815 
bioRxiv preprint doi: https://doi.org/10.1101/2021.01.04.425125; this version posted January 5, 2021. The copyright holder for this preprint (which was not certified by peer review) is the author/funder, who has granted bioRxiv a license to display the preprint in perpetuity. It is made available under aCC-BY-NC 4.0 International license.

(a)

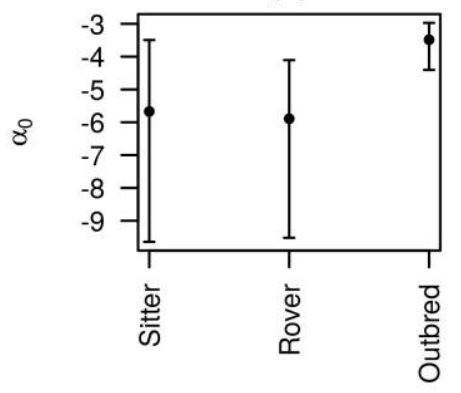

(b)

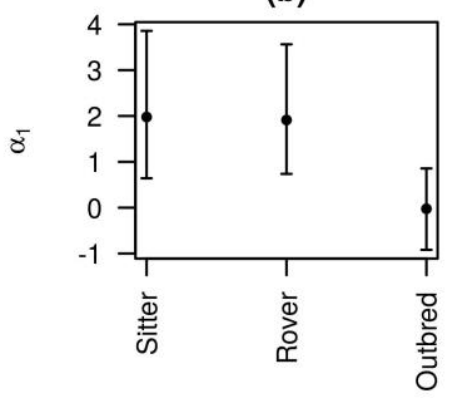

(c)

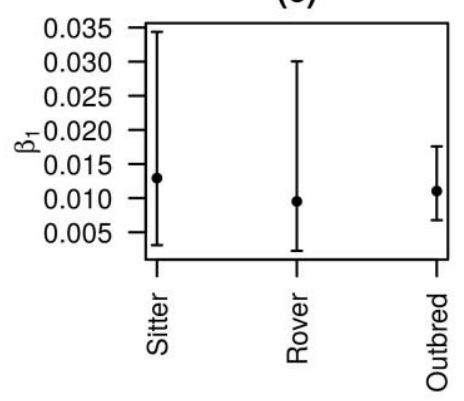

\section{6}

\section{Figure 6.}

\section{8}

819

820

821

822

823

824

825

826

827

828

829

830

831 


\section{$832 \quad$ Figure Captions}

834 Figure 1: (a) The experimental field looking towards the South-West towards Blair Road, Cambridge,

835 ON, Canada. The site is lined by trees on all four sides. (b) Depicting the arrangement of sampling 836 points within the experimental field (blue dots). Values on the $\mathrm{X}$ and $\mathrm{Y}$-axes represent arbitrary 837 coordinates within a $27 \times 27$ matrix with coordinate $(0,0)$ representing the centre. The dotted lines 838 radiating out from the centre represent 16 compass directions corresponding to slices where wind 839 directions were obtained (cardinal directions are not specified on this diagram). The red polygon 840 represents the outline of the $21 \times 21$ matrix that was measured in the field (see red polygon in c). The 841 position of the centre is off-set to increase sampling density in the direction of prevailing winds (see 842 text for more information). (c) Aerial image showing the outline of the experimental field. Each red 843 line along the square depicts the outer trap lines of each side of the sampling grid. The black and red 844 place markers along the red diagonal represent the central release location (e.g. coordinate 0,0$)$ and the 845 weather station that was used to record weather parameters every 15 minutes respectively. (d) Diagram 846 of the traps installed at sample locations. Fly not drawn to scale. See text for more detailed description.

848 Figure 2: Starting from the top of the panel to the bottom we show time series of fly captures across 849 the experimental field. Each square represents a time unique sequence. $t=0.5, t=1.5, t=21, t=26, t=45$ 850 and $\mathrm{t}=50$ represent captures at 30 minutes, $1.5 \mathrm{hr}, 21 \mathrm{hrs}, 26 \mathrm{hrs}, 45 \mathrm{hrs}$ and $50 \mathrm{hrs}$ after release of the 851 flies respectively. The left set of panels represent the time series for sitter $\left(\right.$ for $\left.^{\mathrm{s}}\right)$ flies and the middle and 852 right set of panels represent the time series for rover $\left(\right.$ for $\left.^{\mathrm{R}}\right)$ and outbred flies respectively. The 853 abundance of flies at each sample location are indicated by unique colour coding. Dark blue areas, as 854 indicated by the draped legend on the right, indicate samples with zero fly captures and areas with 855 darkest red indicate samples with abundances of maximum 300 flies. The gray area within each square 856 indicate locations that did not contain traps. 
857 Figure 3: Temperature (a) and wind speed (b) and wind direction (c) measured every 15 minute (y858 axes) over the 50 hours after release (x-axes). Each dot represents a temperature/wind speed/wind 859 direction measurement, blue dots were measured during daylight and black dots represent 860 measurements after daylight. The green vertical dashed lines in (a) (b) and (c) represent the temperature 861 and wind measurements during the six sampling time points.

863 Figure 4: The relationship between temperature (x-axis) and the rate of movement (y-axis) for each fly 864 strain. Each fly strain is represented by a bold line (blue $=$ rovers, for $^{\mathrm{R}}$, black $=$ sitters, for $^{\mathrm{s}}$ and red $=$ 865 outbred flies). The correspondingly coloured shaded areas surrounding each bold line represents the $95 \%$ credible intervals for each fly strain. The thin vertical lines at the bottom of the $\mathrm{x}$-axis indicate the range of temperatures that were measured during the course of this field experiment. At cooler temperatures the outbred strain tended to move faster than the rover and sitter strains, which showed very little movement. At warmer temperatures the diffusion rate of outbred flies remain largely unchanged while rover and sitter diffusion increased (i.e. at temperatures $>18{ }^{\circ} \mathrm{C}$ ).

872 Figure 5: Depicting the relationship between wind speed (x-axis) and advection rate (y-axis). The 873 advection rate (metres per minute) is positive if flies are dispersing in the direction of wind and 874 negative if flies are dispersing against wind direction. For each fly strain the posterior means are represented by bold lines $\left(\right.$ blue $=$ rovers, $f o r^{\mathrm{R}}$, black $=$ sitters, for $^{\mathrm{s}}$ and red $=$ outbred flies $)$. The correspondingly coloured shaded areas surrounding each bold line represents the $95 \%$ credible intervals for each fly strain. For all three strains the advection rate is positive and increases with wind speed. 
880 Figure 6: Parameter estimates for each fly strain associated with diffusion rate (Eq. 2, panels a and b),

881 advection rate (Eq. 3, panel c. Each black dot represents the posterior mean with $95 \%$ credible interval 882 (vertical whiskers). In panel c positive values indicate the tendency to disperse with wind and negative 883 values indicate the tendency to move against wind direction. The advection parameter is positive for all 884 three fly strains. 